\title{
Cumulative association of eight susceptibility genes with systemic lupus erythematosus in a Japanese female population
}

\author{
Minori Koga ${ }^{1,7}$, Aya Kawasaki ${ }^{1,7}$, Ikue Ito ${ }^{1}$, Takumi Furuya ${ }^{1}$, Jun Ohashi ${ }^{1}$, Chieko Kyogoku ${ }^{2}$, Satoshi Ito ${ }^{3,4}$, \\ Taichi Hayashi ${ }^{3}$, Isao Matsumoto ${ }^{3}$, Makio Kusaoi ${ }^{5}$, Yoshinari Takasaki ${ }^{5}$, Hiroshi Hashimoto ${ }^{6}$, \\ Takayuki Sumida ${ }^{3}$ and Naoyuki Tsuchiya ${ }^{1}$
}

\begin{abstract}
Although large-scale studies established many susceptibility genes to systemic lupus erythematosus (SLE), effect of each gene is not sufficiently large to be used alone to identify individuals with strong genetic predisposition. In this study, we analyzed the cumulative number of risk alleles at eight established susceptibility loci, HLA-DRB1, IRF5, STAT4, BLK, TNFAIP3, TNIP1, FCGR2B and TNFSF13, in 282 Japanese female SLE and 222 healthy female controls. The average number of risk alleles was significantly increased in SLE $(8.07 \pm 1.60)$ than healthy controls $(7.02 \pm 1.64)\left(P=1.63 \times 10^{-12}\right)$. Significant gene-gene interaction was not detected. When the subjects carrying seven risk alleles were used as a reference, the odds ratio (OR) for individuals carrying 10 and $11-13$ risk alleles were $4.17(95 \%$ confidence interval $(\mathrm{Cl}) 1.89-9.19, P=0.0002)$ and $8.77(95 \% \mathrm{Cl}$ 1.92-40.0, $P=0.0016)$, respectively. In contrast, subjects with $\leqslant 4$ risk alleles were significantly decreased in SLE (OR 0.15 , $\mathrm{Cl} 0.03-0.67, P=0.007)$. The proportion of the patients with neurologic disorder was significantly increased in those carrying $\geqslant 10$ risk alleles than those with $<10$ (OR $2.30, \mathrm{Cl} 1.09-4.83, P=0.025)$. This study suggested that the cumulative number of risk alleles may efficiently distinguish groups with high and low genetic predisposition to SLE and its severe manifestation. Journal of Human Genetics (2011) 56, 503-507; doi:10.1038/jhg.2011.49; published online 12 May 2011
\end{abstract}

Keywords: genetic risk score; polymorphism; susceptibility; systemic lupus erythematosus

\section{INTRODUCTION}

Recent large-scale association studies identified a number of novel susceptibility genes to systemic lupus erythematosus (SLE), ${ }^{1-5}$ among which many have been replicated in independent studies. However, genetic effect of each susceptibility allele is generally modest; with the exception of HLA-DRB1, none reached the allelic odds ratio (OR) of 2. Thus, the effect of each susceptibility allele is not sufficient to identify the high-risk individuals when used alone.

One possible approach to solve this problem is to combine multiple risk allele genotypes of each individual into a genetic risk score. Such an approach has been employed in type 2 diabetes, ${ }^{6,7}$ cholesterol levels and cardiovascular diseases, ${ }^{8,9}$ prostate cancer ${ }^{10}$ and rheumatic diseases such as gout, ${ }^{11}$ rheumatoid arthritis ${ }^{12}$ and systemic sclerosis. ${ }^{13}$

Our group has been intensively working on the susceptibility genes to SLE in a Japanese population. Thus far, we replicated association of HLA-DRB1 ${ }^{\star} 15: 01$, STAT4 rs7574865T, BLK rs13277113A, TNFAIP3
rs2230926G and TNIP1 rs7708392C in Japanese, which had previously been reported in the Caucasian or Chinese populations. ${ }^{14-18}$ In the process of these studies, we noted that the genetic contribution of STAT4, BLK and TNIP1 was greater in the Japanese compared with the Caucasian populations, due to population differences in the risk allele frequencies. ${ }^{15,16,18}$

With respect to IRF5, we found that the initially reported Caucasian risk haplotype carrying rs2004640T, exon 6 insertion and rs $10954213 \mathrm{~A}^{19}$ was nearly absent in Japanes $\mathrm{e}^{20}$ Moreover, although the association of rs2004640 was also observed in Japanese, another single nucleotide polymorphism (SNP) rs41298401, which is monomorphic in Caucasians, was more strongly associated with SLE. ${ }^{20}$

In FCGR2B, we identified association of a SNP rs1050501, which alters Ile at position 232 to $\mathrm{Thr}$ in the transmembrane region. ${ }^{21}$ This association was replicated in multiple Asian populations. ${ }^{22-24}$ This amino acid substitution was associated with reduced localization of

${ }^{1}$ Molecular and Genetic Epidemiology Laboratory, Doctoral Program in Biomedical Sciences, Graduate School of Comprehensive Human Sciences, University of Tsukuba, Tsukuba, Japan; ${ }^{2}$ Department of Clinical Pathology and Immunology, Kobe University Graduate School of Medicine, Kobe, Japan; ${ }^{3}$ Division of Clinical Immunology, Doctoral Program in Clinical Sciences, Graduate School of Comprehensive Human Sciences, University of Tsukuba, Tsukuba, Japan; ${ }^{4}$ Department of Rheumatology, Niigata Rheumatic Center, Shibata, Japan; ${ }^{5}$ Division of Rheumatology, Department of Internal Medicine, Juntendo University, Tokyo, Japan and ${ }^{6}$ Juntendo University School of Medicine, Tokyo, Japan

${ }^{7}$ These authors contributed equally to this work.

Correspondence: Dr N Tsuchiya, Doctoral Program in Biomedical Sciences, Graduate School of Comprehensive Human Sciences, University of Tsukuba, 1-1-1 Tennodai, Tsukuba, Ibaraki 305-8575, Japan.

E-mail: tsuchiya-tky@umin.ac.jp

Received 13 December 2010; revised 15 April 2011; accepted 18 April 2011; published online 12 May 2011 
inhibitory Fc $\gamma$ receptor IIb molecule in the lipid raft, thereby resulting in hyperactivation of B cells ${ }^{25}$ and monocytes. ${ }^{26}$

In the case of TNFSF13 (APRIL), Koyama et al. ${ }^{27}$ and our group ${ }^{28}$ independently demonstrated association of rs11552708 (Gly67Arg) with SLE in Japanese. This association was also replicated in a study on mixed European-American, African-American and Hispanic populations. ${ }^{29}$ Thus, TNFSF13 is also an established susceptibility gene to SLE.

All of the above mentioned eight susceptibility genes have been independently replicated, and ORs of the risk genotypes are greater than 1.3 in Japanese. ${ }^{14-18,20,21,28}$ In the case of SLE, a recent study from the United States reported association of cumulative risk allele number at 19 susceptibility loci with the age of onset among the patients; ${ }^{30}$ however, association with genetic risk for the occurrence of SLE as compared with healthy controls was not analyzed.

In this study, we combined the genotype data at the eight susceptibility loci established in the Japanese, and tested the association of the cumulative risk allele number with susceptibility to SLE. When the subjects carrying seven risk alleles were used as a reference, the OR for individuals carrying 10 and 11-13 risk alleles were 4.17 and 8.77, respectively. In contrast, subjects with four or less risk alleles were significantly decreased in SLE (OR 0.15). In addition, the proportion of the patients with neurologic disorder was significantly increased in those carrying $\geqslant 10$ risk alleles than those with $<10$. Thus, this study suggested that the cumulative number of risk alleles could potentially be useful for distinguishing groups with high and low genetic predisposition to SLE and its severe manifestation.

\section{MATERIALS AND METHODS}

\section{Patients and controls}

A total of 282 Japanese female patients with SLE and 222 healthy female controls were recruited at University of Tsukuba, Juntendo University and the University of Tokyo. To exclude potential bias caused by gender-associated difference in the genetic factors for SLE, male patients and controls were excluded from this study. All patients and healthy individuals were native Japanese living in the central part of Japan. All patients fulfilled the American College of Rheumatology criteria for SLE. ${ }^{31}$ Presence or absence of renal and neurologic disorders was also classified based on the American College of Rheumatology criteria. ${ }^{31}$ This study was reviewed and approved by the research ethics committees of University of Tsukuba, Juntendo University and the University of Tokyo. Informed consent was obtained from all study participants. This study was conducted in accordance with the principles expressed in the Declaration of Helsinki.

\section{Genotyping}

Genotyping of HLA-DRB1 was performed by PCR-microtiter plate hybridization (MPH) technique or by Luminex Multi-Analyte Profiling system (xMAP) with WAKFlow HLA typing kit (Wakunaga, Hiroshima, Japan) as described elsewhere. ${ }^{32}$ IRF5 rs41298401 genotype was determined by direct sequencing. ${ }^{20}$ FCGR2B rs1050501 was genotyped using nested PCR and oligonucleotide hybridization as previously described, ${ }^{21}$ or direct sequencing using the sequencing primer annealing at intron 5 ( $5^{\prime}$-AATACGGGCCTAGATCTGAATGTG-3') described by Li et al. ${ }^{22}$ after PCR using primers placed at exon 4 (5'-AAGGACA AGCCTCTGGTCAA-3') and exon 7 (5'-CCCAACTTTGTCAGCCTCAT- $\left.3^{\prime}\right){ }^{21}$ TNFSF13 rs11552708 was genotyped using PCR-single strand conformation polymorphism or direct sequencing, as previously described. ${ }^{28}$ Other SNPs were genotyped using the TaqMan genotyping assay (Applied Biosystems, Foster City, CA, USA), as previously described. ${ }^{15-18}$ Identity of the genotyping results were confirmed when two genotyping systems were used for a locus.

The genotyping data of the cases and controls were derived from our previous studies. ${ }^{14-18,20,21,28}$ In addition, when necessary, additional genotyping was performed for individuals in whom any of the eight loci had not been genotyped.

\section{Statistical analysis}

Association of each susceptibility allele with SLE in the cases and controls analyzed this study was performed by Fisher's exact test using $3 \times 2$ contingency table. Gene-gene interaction was examined between each pair of genes using logistic regression analysis under the co-dominant model, where risk allele homozygotes $x_{i}=2$, heterozygotes $x_{i}=1$ and non-risk allele homozygotes $x_{i}=0$ for gene $i$. The logistic regression model for interaction between gene $i$ and gene $j$ was given by

$$
\operatorname{logit}(P)=\beta_{0}+\beta_{i} x_{i}+\beta_{j} x_{j}+\beta_{i j} x_{i} x_{j} .
$$

The deviation from 0 was evaluated for $\beta_{i j}$ by the Wald test.

The increase in the risk by carrying an additional risk allele was calculated using logistic regression analysis. The risk allele count and the disease status were used as independent and dependent variables, respectively.

The statistical significance of the difference in the average number of the risk alleles in cases and controls was examined using Student's $t$-test (two-tailed). When the OR and 95\% confidence interval (CI) was calculated for individuals carrying different number of risk alleles, subjects carrying seven alleles, the median of the risk allele number in healthy controls, were used as a reference. The statistical significance was analyzed using Fisher's exact test.

The proportion of the patients with renal disorder, neurologic disorder or early onset ( $<20$ years) was compared between those with $\geqslant 10$ and $<10$ risk alleles using $\chi^{2}$-test.

\section{RESULTS}

Association between cumulative risk allele number and susceptibility to SLE

We selected eight established susceptibility alleles, $H L A-D R B 1^{\star} 15: 01$, IRF5 rs41298401C, STAT4 rs7574865T, BLK rs13277113A, TNFAIP3 rs2230926G, TNIP1 rs7708392C, FCGR2B rs1050501C and TNFSF13 rs11552708G, based on the following criteria: (1) at least one report of significant association in Japanese, (2) OR of the risk genotype $>1.3$ and (3) at least one independent replication.

These alleles were genotyped in 282 Japanese female patients with SLE and 222 healthy female controls. Table 1 shows the genotype frequencies of the examined loci in the patients and healthy controls. To exclude potential influence from gender-associated difference in the genetic background, we focused on female patients and controls. As a result, the sample size of this study was smaller than our previous studies where each locus was examined individually. ${ }^{14-18,20,21,28}$ This resulted in lack of statistically significant association of TNFAIP3, TNIP1 and TNFSF13 in the subjects analyzed here.

The gene-gene interaction was examined between each pair of two loci (28 pairs) using logistic regression analysis under a co-dominant

Table 1 Genotype frequencies of each susceptibility gene with systemic lupus erythematosus in the Japanese female patients and controls examined in this study

\begin{tabular}{|c|c|c|c|c|c|c|c|c|}
\hline \multirow[b]{2}{*}{ Locus } & \multirow[b]{2}{*}{ Risk allele } & \multicolumn{3}{|c|}{ SLE (\%) } & \multicolumn{3}{|c|}{ Healthy controls (\%) } & \multirow[b]{2}{*}{$\mathrm{P}$} \\
\hline & & 11 & 12 & 22 & 11 & 12 & 22 & \\
\hline$H L A-D R B 1$ & *15:01 & 3.2 & 22.0 & 74.8 & 1.4 & 8.1 & 90.5 & $1.37 \times 10^{-5}$ \\
\hline IRF5 & rs41298401C & 73.8 & 25.9 & 0.4 & 60.8 & 35.1 & 4.1 & $3.71 \times 10^{-4}$ \\
\hline STAT4 & rs7574865T & 18.4 & 55.7 & 25.9 & 11.7 & 45.9 & 42.3 & $3.20 \times 10^{-4}$ \\
\hline$B L K$ & rs13277113A & 62.8 & 29.1 & 8.2 & 46.8 & 42.8 & 10.4 & $1.55 \times 10^{-3}$ \\
\hline TNFAIP3 & rs2230926G & 1.8 & 17.4 & 80.9 & 0.5 & 16.2 & 83.3 & 0.41 \\
\hline$T N I P 1$ & rs7708392C & 58.9 & 34.4 & 6.7 & 51.4 & 40.5 & 8.1 & 0.24 \\
\hline FCGR2B & rs1050501C & 10.3 & 36.5 & 53.2 & 4.1 & 38.3 & 57.7 & 0.03 \\
\hline TNFSF13 & rs11552708G & 39.7 & 48.6 & 11.7 & 36.9 & 47.7 & 15.3 & 0.48 \\
\hline
\end{tabular}

Genotype frequencies of the tested eight susceptibility genes in Japanese female systemic lupus erythematosus patients $(n=282)$ and healthy controls $(n=222)$ are examined in this study. $P$ values were calculated by Fisher's exact test using $2 \times 3$ contingency tables. $1=$ risk allele, $2=$ non-risk allele. 
model. Statistically significant gene-gene interaction was not observed, except for a marginal interaction between $B L K$ and FCGR2B (uncorrected $P=0.04$ ).

Table 2 shows the distribution of the cumulative risk allele number of each subject in the patients and healthy controls. Risk allele number was distributed between 4-13 in the patients and 3-11 in the controls. The average number of the risk alleles was significantly increased in the patients $(8.07 \pm 1.60$ alleles $)$ as compared with controls (7.02 \pm 1.64 alleles $)\left(P=1.63 \times 10^{-12}\right.$, Student's $t$-test $)$.

Figure 1 shows the OR and $95 \%$ CI for each group with increasing number of risk alleles, as compared with the individuals carrying seven risk alleles, the median risk allele count in the control group. In this analysis, groups with 11-13 and 3-4 risk alleles were combined due to the small number of the subjects in each group (Table 2). Because

Table 2 Numbers and percentages of the patients and healthy controls carrying each cumulative risk allele number

\begin{tabular}{lcc}
\hline Number of risk alleles & $S L E(\mathrm{n}=282)$ & Control $(\mathrm{n}=222)$ \\
\hline 3 & $0(0)$ & $6(2.7)$ \\
4 & $2(0.7)$ & $9(4.1)$ \\
5 & $14(5.0)$ & $24(10.8)$ \\
6 & $30(10.6)$ & $39(17.6)$ \\
7 & $52(18.4)$ & $57(25.7)$ \\
8 & $78(27.7)$ & $46(20.7)$ \\
9 & $52(18.4)$ & $29(13.1)$ \\
10 & $38(13.5)$ & $10(4.5)$ \\
11 & $11(3.9)$ & $2(0.9)$ \\
12 & $4(1.4)$ & $0(0)$ \\
13 & $1(0.4)$ & $0(0)$ \\
\hline
\end{tabular}

Abbreviation: SLE, systemic lupus erythematosus.

Percentages are shown in parentheses.

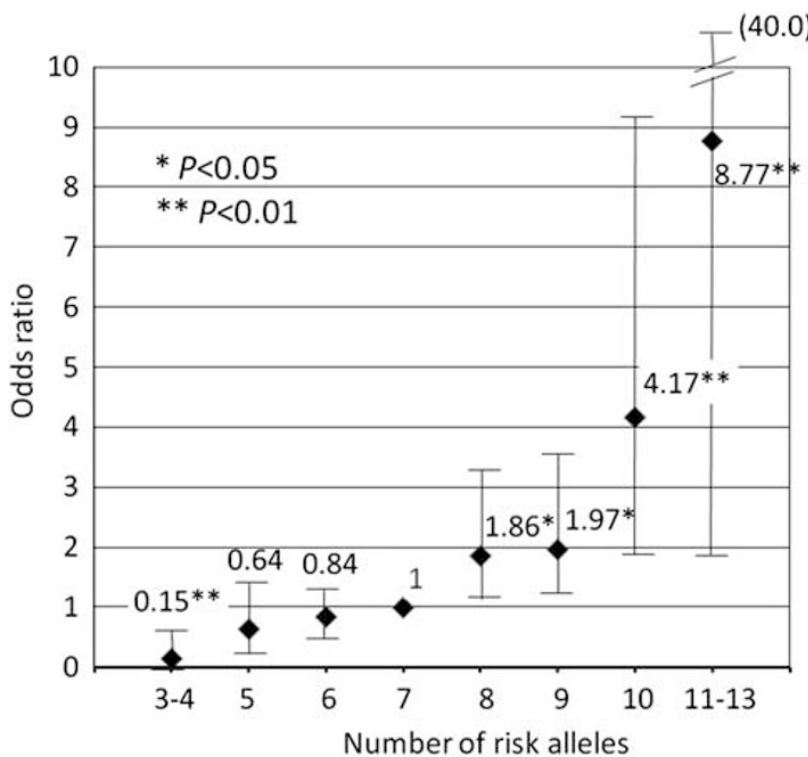

Figure 1 Odds ratios and 95\% confidence intervals of the individuals carrying increasing numbers of risk alleles in eight SLE susceptibility loci. The reference group was the individuals with seven risk alleles, which was the median of the healthy controls. Statistical significance was tested using Fisher's exact test. Power calculation using computer simulation (1000 runs) demonstrated that the power to detect statistical significance at the significance level of 0.05 was $87.4 \%$ (3-4 alleles), $26.8 \%$ ( 5 alleles), $13.0 \%$ (6 alleles), 69.3\% (8 alleles), 67.9\% (9 alleles), 98.3\% (10 alleles) and $94.9 \%$ (11-13 alleles), respectively. apparent gene-gene interaction was not observed, the increase in the risk by carrying an additional risk allele was estimated using logistic regression analysis, where the risk allele count and the disease status were used as independent and dependent variables, respectively. This analysis indicated that each additional risk allele increased the OR by 1.50 fold (95\% CI: $\left.1.33-1.69, P=4.5 \times 10^{-11}\right)$.

When compared with individuals carrying seven risk alleles, those with eight or more risk alleles was associated with significantly elevated OR $(P<0.05$, Fisher's exact test) (Figure 1). Specifically, the ORs of individuals with 10 and 11-13 risk alleles were 4.17 (CI 1.899.19, $P=0.0002$ ) and 8.77 (CI 1.92-40.0, $P=0.0016$ ), respectively.

On the other hand, the OR in individuals having four or less risk alleles was significantly decreased in SLE (OR 0.15, CI 0.03-0.67, $P=0.0070$ ). When individuals carrying 11 or more risk alleles were compared with those carrying four or less alleles, the OR was 60 (CI 7.48-481.6, $P=5.2 \times 10^{-6}$ ).

Power calculation was performed using computer simulation (1000 runs). This sample size provided $95.7 \%$ (seven versus eight or more alleles), $98.3 \%$ (seven versus 10 alleles), 94.9\% (seven versus $11-13$ alleles), $87.4 \%$ (seven versus 3-4 alleles) and 99.8\% (3-4 versus $11-13$ alleles) power to detect statistical difference at the significance level of 0.05 .

\section{Association between cumulative risk allele number and clinical subsets}

We next examined whether the cumulative risk allele number is associated with severe clinical phenotypes such as renal disorder and neurologic disorder, as well as with early onset of SLE. First, we compared the mean risk allele number between the patients with and without each clinical feature. The mean number of risk alleles showed a tendency towards increase in the patients with renal disorder $(8.21 \pm 1.57$ alleles, $n=156)$ as compared with those without $(7.90 \pm 1.64$ alleles, $n=125)$, in those with neurologic disorder $(8.39 \pm 1.96, n=41)$ than those without $(8.02 \pm 1.53, n=232)$ and in the patients with early onset ( $<20$ years) $(8.10 \pm 1.42, n=72)$ than those with later onset ( $\geqslant 20$ years) $(8.07 \pm 1.66, n=208)$, although none of the differences reached statistical significance ( $P>0.05$, Student's $t$-test).

We then compared the proportion of each clinical characteristics between the patients carrying $\geqslant 10$ risk alleles and those with $<10$. As shown in Table 3, significant increase of neurologic disorder was observed in the patients carrying $\geqslant 10$ risk alleles.

Table 3 Comparison of clinical phenotypes between SLE patients with $\geqslant 10$ and $<10$ risk alleles

\begin{tabular}{|c|c|c|c|c|}
\hline & $\begin{array}{c}\geqslant 10 \text { risk alleles } \\
\mathrm{n}(\%)\end{array}$ & $\begin{array}{c}<10 \text { risk alleles } \\
\mathrm{n}(\%)\end{array}$ & P & OR $(95 \% \mathrm{Cl})$ \\
\hline \multicolumn{5}{|c|}{ Age of onset } \\
\hline$<20$ & $11(20.4)$ & $61(27.0)$ & 0.317 & $0.69(0.34-1.43)$ \\
\hline$\geqslant 20$ & $43(79.6)$ & $165(73.0)$ & & \\
\hline \multicolumn{5}{|c|}{ Renal disorder } \\
\hline+ & $35(64.8)$ & $121(53.3)$ & 0.126 & $1.61(0.87-2.99)$ \\
\hline- & $19(35.2)$ & $106(46.7)$ & & \\
\hline \multicolumn{5}{|c|}{ Neurologic disorder } \\
\hline+ & $13(25.0)$ & $28(12.7)$ & 0.025 & $2.30(1.09-4.83)$ \\
\hline- & $39(75.0)$ & 193 (87.3) & & \\
\hline
\end{tabular}

Abbreviations: $\mathrm{Cl}$, confidence interval; OR, odds ratio; SLE, systemic lupus erythematosus. $P$ values were calculated by $\chi^{2}$-test. Power calculation using computer simulation (1000 runs) revealed that this sample size provided $20.9 \%$ (age of onset), $36.8 \%$ (renal disorder) and $61.4 \%$ (neurologic disorder) power to detect statistical difference at the significance level of 0.05 . 


\section{DISCUSSION}

Attempts to develop a marker for estimating the risk of an individual by using cumulative risk allele number at multiple loci have been reported in various complex diseases. ${ }^{6-13}$ In this study, by combining the number of risk alleles at eight susceptibility loci, each having rather small allelic OR, it became possible to distinguish high and low risk groups with up to 60 -fold difference in the genetic predisposition to SLE. However, it should be noted that the model proposed in this study is, at this point, not sufficient for the purpose of predicting disease onset or identifying a high-risk group that requires preventive intervention. This is because of the low incidence of SLE, lack of measures for prevention, as well as the limitations of the study approach as described later.

In this study, we simply counted the number of risk alleles of multiple susceptibility loci, Our model could potentially be improved by weighting each risk allele according to the size of genetic effects (ORs) as described in the study of RA, ${ }^{12}$ or taking the appropriate genetic model (for example dominant, co-dominant or recessive) into account. However, the genetic effect of each allele and the model that provides the best fit sometimes vary considerably among studies. In addition, in most of the susceptibility genes, the causative allele, which accounts for the molecular mechanism of SLE has yet to be identified. The ORs of the unidentified causative alleles may be different from those observed for the 'proxy' SNPs. Therefore, we employed a modelfree approach to simply count the number of risk alleles. Our data suggest that even this simple approach could potentially provide a useful parameter to estimate genetic predisposition to SLE of each individual. With establishment of many more susceptibility genes, this model is expected to improve its power to identify individuals with elevated genetic risk to develop SLE.

There are several limitations in this study. Because of the low prevalence of SLE, the sample size of this study is small, which resulted in the wide range of CIs in the subjects carrying 10 or more risk alleles. Although significant gene-gene interaction was not detected in every possible combination of two genes, it would be intriguing to examine the combined effects of specific risk genotypes at three or more susceptibility loci. However, such a study requires much larger number of subjects than this study. For these reasons, we admit that this study is somewhat preliminary and the results should be validated in a larger sample set.

One approach to increase the sample size in the study of rare diseases is to organize a large-scale international collaboration. However, we focused on the Japanese population, because we wanted to find a biomarker useful in clinical practice or preventive medicine in Japanese. The genetic background has been shown to be slightly different even among the closely-related East Asian populations. ${ }^{33,34}$ Therefore, the next step will be to validate our results in a larger sample size through collaboration with multiple SLE centers in Japan.

Another possibility is the use of GWAS data of Japanese SLE. Although GWAS of Japanese SLE has not been reported yet, once the data become available, it might be possible to impute the genotypes at the susceptibility genes analyzed in this study to compare cumulative risk allele numbers between a large number of patients and controls.

It should be noted that the set of eight genes in this study was based on the Japanese data, and is not directly applicable to other populations. For example, the risk alleles of PTPN22 $2^{35}$ and ITGAM ${ }^{1,2}$ as well as $H L A-D R B 1^{*} 03: 01,{ }^{36}$ established risk factors for SLE in the Caucasian populations, are absent or very rare in the Japanese population. Thus, similar analysis should be carried out in each population, using the set of established susceptibility genes in the target population.
It should also be emphasized that this study considered only the SLE susceptibility genes that have already been replicated in the Japanese population. Recently reported SLE susceptibility genes, which have yet to be replicated in Japanese such as TNFSF4, $4,5,37$ were not included. For the same reason, we did not include the multiple SNPs in the HLA region, which were shown to be independently associated with SLE in other populations. ${ }^{38}$ Therefore, our current model represents only a proportion of the whole genetic contribution to SLE, and should be improved in the future by incorporating additional susceptibility genes when their association was replicated in the Japanese.

The cumulative risk allele number might potentially be useful for the prediction of disease phenotype among the patients. In a recent study in the United States, the risk allele number was associated with early onset of SLE, which in turn was associated with severe disease. ${ }^{30}$ In our data, the association between the risk allele number and early onset was not detected. However, of particular interest, the patients carrying $\geqslant 10$ risk alleles exhibited more than twofold risk for developing neurologic disorder, one of the severe manifestations of the disease, than those with 10 risk alleles. Such information would be useful for personalized medicine; for example, more aggressive treatment may be considered for the patients with increased number of risk alleles.

The cumulative risk allele number may also be useful for research purpose. For example, longitudinal studies of healthy individuals carrying an increased number of risk alleles may efficiently identify environmental triggers or epigenetic modifiers that lead to the development of SLE. It would also be of interest to test association with the presence of antinuclear antibodies among healthy individuals. ${ }^{39}$

\section{ACKNOWLEDGEMENTS}

This work was supported by Grant-in-Aid for Scientific Research (B) 22390199 and Grant-in-Aid for Young Scientists (B) 21790935 from Japan Society for the Promotion of Science (JSPS), Health and Labour Science Research Grants for the Research on Intractable Diseases from the Ministry of Health, Labour and Welfare of Japan, the Takeda Science Foundation and Japan Rheumatism Foundation.

1 Hom, G., Graham, R. R., Modrek, B., Taylor, K. E., Ortmann, W., Garnier, S. et al. Association of systemic lupus erythematosus with C8orf13-BLK and ITGAM-ITGAX. N. Engl. J. Med. 358, 900-909 (2008).

2 International Consortium for Systemic Lupus Erythematosus Genetics (SLEGEN), Harley, J. B., Alarcón-Riquelme, M. E., Criswell, L. A., Jacob, C. O., Kimberly, R. P., Moser, K. L. et al. Genome-wide association scan in women with systemic lupus erythematosus identifies susceptibility variants in ITGAM, PXK, KIAA1542 and other loci. Nat. Genet. 40, 204-210 (2008).

3 Gateva, V., Sandling, J. K., Hom, G., Taylor, K. E., Chung, S. A., Sun, X. et al. A largescale replication study identifies TNIP1, PRDM1, JAZF1, UHRF1BP1 and ILIO as risk loci for systemic lupus erythematosus. Nat. Genet. 41, 1228-1233 (2009).

4 Han, J. W., Zheng, H. F., Cui, Y., Sun, L. D., Ye, D. Q., Hu, Z. et al. Genome-wide association study in a Chinese Han population identifies nine new susceptibility loci for systemic lupus erythematosus. Nat. Genet. 41, 1234-1237 (2009).

5 Yang, W., Shen, N., Ye, D. Q., Liu, Q., Zhang, Y., Qian, X. X. et al. Genome-wide association study in Asian populations identifies variants in ETS1 and WDFY4 associated with systemic lupus erythematosus. PLoS Genet. 6, e1000841 (2010).

6 Weedon, M. N., McCarthy, M. I., Hitman, G., Walker, M., Groves, C. J., Zeggini, E. et al. Combining information from common type 2 diabetes risk polymorphisms improves disease prediction. PLoS Med. 310, e374 (2006).

7 Miyake, K., Yang, W., Hara, K., Yasuda, K., Horikawa, Y., Osawa, H. et al. Construction of a prediction model for type 2 diabetes mellitus in the Japanese population based on 11 genes with strong evidence of the association. J. Hum. Genet. 54, 236-241 (2009).

8 Kathiresan, S., Melander, O., Anevski, D., Guiducci, C., Burtt, N. P., Roos, C. et al. Polymorphisms associated with cholesterol and risk of cardiovascular events. N. Engl. J. Med. 358, 1240-1249 (2008).

9 Anderson, J. L., Horne, B. D., Camp, N. J., Muhlestein, J. B., Hopkins, P. N., CannonAlbright, L. A. et al. Joint effects of common genetic variants from multiple genes 
and pathways on the risk of premature coronary artery disease. Am. Heart J. 160, 250-256.e3 (2010).

10 Zheng, S. L., Sun, J., Wiklund, F., Smith, S., Stattin, P., Li, G. et al. Cumulative association of five genetic variants with prostate cancer. N. Engl. J. Med. 358, 910-919 (2008).

11 Dehghan, A., Köttgen, A., Yang, Q., Hwang, S. J., Kao, W. L., Rivadeneira, F. et al. Association of three genetic loci with uric acid concentration and risk of gout: a genome-wide association study. Lancet 372, 1953-1961 (2008).

12 Karlson, E. W. K., Chibnik, L. B., Kraft, P., Cui, J., Keenan, B. T., Ding, B. et al. Cumulative association of 22 genetic variants with seropositive rheumatoid arthritis risk. Ann. Rheum. Dis. 69, 1077-1085 (2010).

13 Dieudé, P., Wipff, J., Guedj, M., Ruiz, B., Melchers, I., Hachulla, E. et al. BANK1 is a genetic risk factor for diffuse cutaneous systemic sclerosis and has additive effects with IRF5 and STAT4. Arthritis Rheum. 60, 3447-3454 (2009).

14 Tsuchiya, N., Ohashi, J. \& Tokunaga, K. Variations in immune response genes and their associations with multifactorial immune disorders. Immunol. Rev. 190, 169-181 (2002).

15 Kawasaki, A., Ito, I., Hikami, K., Ohashi, J., Hayashi, T., Goto, D. et al. Role of STAT4 polymorphisms in systemic lupus erythematosus in a Japanese population: a case-control association study of the STAT1-STAT4 region. Arthritis Res. Ther. 10, R113 (2008).

16 Ito, I., Kawasaki, A., Ito, S., Hayashi, T., Goto, D., Matsumoto, I. et al. Replication of the association between C8orf13-BLK region and systemic lupus erythematosus in a Japanese population. Arthritis Rheum. 60, 553-558 (2009).

17 Kawasaki, A., Ito, I., Ito, S., Hayashi, T., Goto, D., Matsumoto, I. et al. Association of TNFAIP3 polymorphism with susceptibility to systemic lupus erythematosus in a Japanese population. J. Biomed. Biotechnol 2010, 207578 (2010). doi:10.1155/ 2010/207578

18 Kawasaki, A., Ito, S., Furukawa, H., Hayashi, T., Goto, D., Matsumoto, I. et al. Association of TNFAIP3 interacting protein 1, TNIP1 with systemic lupus erythematosus in a Japanese population: a case-control association study. Arthritis Res. Ther. 12, R174 (2010).

19 Graham, R. R., Kyogoku, C., Sigurdsson, S., Vlasova, I. A., Davies, L. R., Baechler, E. C. et al. Three functional variants of IFN regulatory factor 5 (IRF5) define risk and protective haplotypes for human lupus. Proc. Natl. Acad. Sci. USA 104, 6758-6763 (2007).

20 Kawasaki, A., Kyogoku, C., Ohashi, J., Miyashita, R., Hikami, K., Kusaoi, M. et al. Association of IRF5 polymorphisms with systemic lupus erythematosus in a Japanese population. Support for a crucial role of intron 1 polymorphisms.. Arthritis Rheum. 58, 826-834 (2008).

21 Kyogoku, C., Dijstelbloem, H. M., Tsuchiya, N., Hatta, Y., Kato, H., Yamaguchi, A et al. Fc $\gamma$ receptor gene polymorphisms in Japanese patients with systemic lupus erythematosus: contribution of FCGR2B to genetic susceptibility. Arthritis Rheum. 46, 1242-1254 (2002).

$22 \mathrm{Li}$, X., Wu, J., Carter, R. H., Edberg, J. C., Su, K., Cooper, G. S. et al. A novel polymorphism in the $\mathrm{F} c \gamma$ receptor IIB (CD32B) transmembrane region alters receptor signaling. Arthritis Rheum. 48, 3242-3252 (2003).

23 Smith, K. G. C. \& Clatworthy, M. R. Fc $\gamma$ RIIB in autoimmunity and infection: evolutionary and therapeutic implications. Nat. Rev. Immunol. 10, 328-343 (2010).
24 Tsuchiya, N., Honda, Z. \& Tokunaga, K. Role of B cell inhibitory receptor polymorphisms in systemic lupus erythematosus: a negative times a negative makes a positive. J. Hum. Genet. 51, 741-750 (2006).

25 Kono, H., Kyogoku, C., Suzuki, T., Tsuchiya, N., Honda, H., Yamamoto, K. et al. Fc $\gamma$ RIIB Ile232Thr transmembrane polymorphism associated with human systemic lupus erythematosus decreases affinity to lipid rafts and attenuates inhibitory effects on B cell receptor signaling. Hum. Mol. Genet. 14, 2881-2892 (2005).

26 Floto, R. A., Clatworthy, M. R., Heilbronn, K. R., Rosner, D. R., MacAry, P. A., Rankin, A. et al. Loss of function of a lupus-associated Fc $\gamma$ Rllb polymorphism through exclusion from lipid rafts. Nat. Med. 11, 1056-1058 (2005).

27 Koyama, T., Tsukamoto, H., Masumoto, K., Himeji, D., Hayashi, K., Harada, M. et al. A novel polymorphism of the human $A P R / L$ gene is associated with systemic lupus erythematosus. Rheumatology 42, 980-985 (2003).

28 Kawasaki, A., Tsuchiya, N., Ohashi, J., Murakami, Y., Fukazawa, T., Kusaoi, M. et al. Role of APRIL (TNFSF13) polymorphisms in the susceptibility to systemic lupus erythematosus in Japanese. Rheumatology 46, 776-782 (2007).

29 Lee, Y. H., Ota, F., Kim-Howard, X., Kaufman, K. M. \& Nath, S. K. APRIL polymorphism and systemic lupus erythematosus (SLE) susceptibility. Rheumatology 46, 1274-1276 (2007).

30 Webb, R., Kelly, J. A., Somers, E. C., Hughes, T., Kaufman, K. M., Sanchez, E. et al. Early disease onset is predicted by a higher genetic risk for lupus and is associated with a more severe phenotype in lupus patients. Ann. Rheum. Dis. 70 (1), 151-156 (2010).

31 Hochberg, M. C. Updating the American College of Rheumatology revised criteria for the classification of systemic lupus erythematosus. Arthritis Rheum 40, 1725 (1997).

32 Hoa, B. K., Hang, N. T., Kashiwase, K., Ohashi, J., Lien, L. T., Horie, T. et al. HLA-A, -B, $-C,-D R B 1$ and $-D Q B 1$ alleles and haplotypes in the Kinh population in Vietnam. Tissue Antigens 71, 127-134 (2008).

33 Tian, C., Kosoy, R., Lee, A., Ransom, M., Belmont, J. W., Gregersen, P. K. et al. Analysis of East Asia genetic substructure using genome-wide SNP arrays. PLoS One 3, e3862 (2008).

34 Shen, N., Fu, Q , Deng, Y. Qian, X. Zhao, J., Kaufman, K. M. et al. Sex-specific association of X-linked Toll-like receptor 7 (TLRT) with male systemic lupus erythematosus. Proc. Natl. Acad. Sci. USA 107, 15838-15843 (2010).

35 Kyogoku, C., Langefeld, C. D., Ortmann, W. A., Lee, A., Selby, S., Carlton, V. E et al. Genetic association of the R620W polymorphism of protein tyrosine phosphatase PTPN22 with human SLE. Am. J. Hum. Genet. 75, 504-507 (2004).

36 Graham, R. R., Ortmann, W. A., Langefeld, C. D., Jawaheer, D., Selby, S. A., Rodine, P. R. et al. Visualizing human leukocyte antigen class II risk haplotypes in human systemic lupus erythematosus. Am. J. Hum. Genet. 71, 543-553 (2002).

37 Cunninghame Graham, D. S., Graham, R. R., Manku, H., Wong, A. K., Whittaker, J. C., Gaffney, P. M. et al. Polymorphism at the TNF superfamily gene TNFSF4 confers susceptibility to systemic lupus erythematosus. Nat. Genet. 40, 83-89 (2008).

38 Barcellos, L. F., May, S. L., Ramsay, P. P., Quach, H. L., Lane, J. A., Nititham, J. et al. High-density SNP screening of the major histocompatibility complex in systemic lupus erythematosus demonstrates strong evidence for independent susceptibility regions. PLoS Genet. 5, e1000696-456 (2009).

39 Tan, E. M., Feltkamp, T. E., Smolen, J. S., Butcher, B., Dawkins, R., Fritzler, M. J. et al. Range of antinuclear antibodies in 'healthy' individuals. Arthritis Rheum. 40, 1601-1611 (1997). 